\title{
Predictors of periprocedural complications in patients undergoing percutaneous coronary interventions within coronary artery bypass grafts
}

\author{
Rafał A. Januszek ${ }^{1,2}$, Artur Dziewierz ${ }^{2,3}$, Zbigniew Siudak ${ }^{4}$, \\ Tomasz Rakowski ${ }^{2}$, Dariusz Dudek ${ }^{2,3,5}$, Stanisław Bartuśs s, $^{2}$
}

${ }^{1}$ Department of Clinical Rehabilitation, University of Physical Education, Krakow, Poland

${ }^{2} 2^{\text {nd }}$ Department of Cardiology and Cardiovascular Interventions, University Hospital, Krakow, Poland

${ }^{3} 2^{\text {nd }}$ Department of Cardiology, Jagiellonian University Medical College, Krakow, Poland

${ }^{4}$ Faculty of Medicine and Health Sciences, Jan Kochanowski University, Kielce, Poland

${ }^{5}$ Department of Interventional Cardiology, Jagiellonian University Medical College, Krakow, Poland

\begin{abstract}
Background: During the first decade following the coronary bypass grafting, at least ten percent of the patients require percutaneous coronary interventions (PCI) due to graft failure. Saphenous vein grafts $(S V G)$ are innately at a higher risk of periprocedural complications. The present study aimed to investigate predictors of periprocedural complications of PCI within coronary artery bypass grafts.

Methods: This study analyzed data gathered in the Polish National Registry (ORPKI) between January 2015 and December 2016. Of the 221,195 patients undergoing PCI, data on 2,616 patients after PCI of SVG and 442 patients after internal mammary artery (IMA) were extracted. The dissimilarities in periprocedural complications between the SVG, IMA and non-IMA/SVG groups and their predictors were investigated.

Results: Patients in the SVG group were older $(p<0.001)$, with a higher burden of concomitant disease and differing clinical presentation. The rate of de-novo lesions was lower, while restenosis was higher at baseline in the SVG $(p<0.001)$. The rate of no-reflows $(p<0.001)$, perforations $(p=0.01)$ and all periprocedural complications $(p<0.01)$ was higher in the $S V G$ group, while deaths were lower $(p<0.001)$. Among the predictors of no-reflows, it was found that acute coronary syndromes (ACS), thrombectomy and past cerebral stroke, while the complications included arterial hypertension, Thrombolysis in Myocardial Infarction (TIMI) flow before PCI and thrombectomy.

Conclusions: Percutaneous coronary interventions of SVG is associated with increased risk of specific periprocedural complications. The ACS, slower TIMI flow before PCI and thrombectomy significantly increase the periprocedural complication rate in patients undergoing PCI of SVG. (Cardiol J 2019; 26, 6: 633-644)
\end{abstract}

Key words: percutaneous coronary interventions, coronary artery by-passes, periprocedural complications, no-reflow, predictors

\section{Introduction}

Within 10 years of coronary artery bypass graft surgery $(\mathrm{CABG})$, even half of patients can be observed with graft failure [1]. Failed patency is usually caused by graft failure or a combination of graft failure and progression of coronary atherosclerosis. Neointimal hyperplasia is considered

Address for correspondence: Stanisław Bartuś, MD, PhD, $2^{\text {nd }}$ Department of Cardiology, Jagiellonian University Medical College, ul. Kopernika 17, 31-501 Kraków, Poland, tel: +48 12424 71 70, fax: +48 1242471 80,

e-mail: stanislaw.bartus@uj.edu.pl

Received: 8.01.2018 Accepted: 18.03.2018 
to be the main reason for the occlusion of venous grafts [2]. The optimal management of patients presenting with graft failure remains a subject of debate. Surgical reoperation is associated with high complication rates and mortality compared to conventional percutaneous coronary interventions (PCI) [3]. Also, repeat operations are associated with higher morbidity and mortality rates as well as poorer outcomes compared to initial operations [4]. Although endovascular treatment of saphenous vein grafts (SVGs) is connected with better results in comparison to re-CABG, there is still a great gap between PCI performed on native arteries and SVG, which reflects poorer results including higher in-hospital mortality among patients undergoing PCI of SVG [5]. Among several predictors of outcomes, the elevated markers of myocardial injury may translate into increased mortality when compared to patients with native vessel PCI [6]. Other variables which have been associated with increased risk of complications after PCI of SVG lesions include old, diffusely diseased, totally occluded grafts and grafts containing intraluminal thrombus with increased lesion friability and propensity for distal embolization [7].

The aim of the present study was to determine the procedural success, periprocedural complications and predictors of typical complications for SVG PCI in a consecutive series of patients undergoing a non-staged SVG intervention in Poland in 2015 and 2016.

\section{Methods}

\section{Study population, design and definitions}

Data from all patients who underwent PCI in Poland between January 2015 and December 2016 were analyzed. Prospectively collected data on PCI practice in Poland were obtained from the ORPKI Polish National dataset, which is coordinated nationwide by Jagiellonian University Medical College in cooperation with AISN PTK (Association of Cardiovascular Interventions Polish Cardiac Society). Database characteristics and data collection methods have been previously presented [8, 9]. Patients were categorized according to whether they had undergone PCI of SVG or the right/left internal mammary artery (IMA). PCI of all other coronary arteries, except SVGs and IMAs were included into the non-IMA/SVG group. All indices recorded in the ORPKI database are based on periprocedural data uploaded by the operator after each procedure. Therefore, they do not include all in-hospital complications, mainly those which occurred after the procedure until discharge from the hospital. Also, follow-up data after discharge was not collected due to lack of patient ID details. The decision to perform PCI of SVG or IMA was at the operators' discretion at each center according to current guidelines [10]. All clinical decisions, such as vascular access, thrombectomy, treatment with glycoprotein (GP) IIb/IIIa inhibitors or bivalirudin, were at the operators' discretion. The definition of periprocedural complications including death, perforation, dissection, myocardial infarction (MI), allergic reaction, cerebral stroke, puncture site bleeding, no-reflow or cardiac arrest remained to the operators' personal preferences and knowledge [8].

\section{Statistical analysis}

All continuous variables were evaluated with the Kolmogorov-Smirnov test for distribution. Continuous variables are presented as mean \pm \pm standard deviation and median \pm interquartile range. Categorical variables are presented as numeric values and percentages. Continuous variables were compared using the two-tailed Student t-test and the Mann-Whitney U-test, whereas categorical variables used the $\chi^{2}$ test. Statistical significance was accepted at a 0.05 level of probability. The statistical analyses were performed using Statistica 10.0 software (Dell Software, Inc, Round Rock, TX, USA) and SPSS STATISTICS 24 (IBM, USA).

\section{Multifactorial analysis}

Since the periprocedural complication rates in the IMA group of patients did not differ significantly from the non-IMA/SVG group when particular complications were compared, the present analysis concentrated on the comparison of the SVG and the non-IMA/SVG group. To identify predictors of all complications, no-reflows, deaths and perforations, univariate and multivariable analysis was performed. In this analysis, the following variables were tested in the SVG group: age, gender, diabetes, previous cerebral stroke, MI, PCI, CABG, smoking status, concomitant diseases including psoriasis, hypertension, kidney disease, chronic obstructive pulmonary disease, clinical presentation of coronary artery disease (CAD) at baseline (stable angina [SA] vs. acute coronary syndrome [ACS], SA vs. acute MI [AMI], SA vs. unstable angina [UA], UA vs. AMI), pharmacological treatment before PCI and during PCI (acetylsalicylic acid [ASA], unfractionated heparin [UFH], low-molecular weight heparin [LMWH], $\mathrm{P}_{2} \mathrm{Y}_{12}$ inhibitors, 
thrombolysis, GP IIb/IIIa inhibitors, bivalirudin), angiographic presentation of CAD (single-vessel disease [SVD] vs. others, left-main coronary artery [LMCA] involvement vs. others), vascular access (radial vs. femoral), fractional flow reserve (FFR), intravascular ultrasound (IVUS), optical coherent tomography (OCT), thrombectomy, pharmacological treatment, Thrombolysis in Myocardial Infarction (TIMI) flow before PCI (1-2 vs. 2-3), contrast dose and radiation exposition, $\mathrm{PCI}$ of chronic total occlusions (CTO) or bifurcation, stent implantation, drug-eluting stent (DES) implantation, number of implanted DES stents (1 vs. 2 or more stents), bare-metal stent (BMS) implantation, number of implanted stents regardless of type (1 vs. 2 or more stents), bioresorbable scaffold (BRS) implantation, PCI with drug-coated balloon (DCB) and PCI with $\mathrm{DCB} /$ plain old balloon angioplasty (POBA)/failed. Also performed were univariate and multivariable analysis of potential predictors of all complications, no-reflows, deaths and perforations in the overall group of patients. In this analysis, rotablations (RAs), were additionally included.

\section{Results}

\section{General characteristics}

The general characteristics of patients examined in the current study including concomitant diseases, past cardiovascular procedures, gender and age in the assessed groups of patients and according to PCI of SVG and IMA are presented in Table 1.

\section{Clinical presentation, coronary angiography and vascular access}

The clinical presentations of CAD included SA, UA, non-ST-segment elevation MI (NSTEMI), ST-segment elevation MI (STEMI), AMI or others where such indications like cardiac arrest or valvular diseases were included. Coronary angiography was divided into four groups: SVD, multi-vessel disease (MVD) without LMCA involvement, MVD with LMCA involvement and isolated LMCA involvement. Vascular access was assessed as femoral access, right and left radial access and others. All the above-mentioned indices were compared in three separate groups: non-IMA/SVG group, IMA and SVG group and are presented in Table 1.

\section{Procedural variables}

Selected procedural indices were compared which involved procedural contrast dose, radiation exposition, use of FFR, IVUS and OCT, type of le- sion undergoing PCI including CTO, bifurcations, type of the procedure with stent type (BMS, DES, BRS, DCB, POBA or failed PCI) and the use of additional devices such as RA or thrombectomy in three separate groups of patients: non-IMA/SVG group, SVG and IMA group. This is presented in Table 2.

\section{Pharmacological treatment}

Pharmacological treatment was also compared before and during PCI between three selected groups of patients including non-IMA/SVG group, IMA and SVG group, which was presented in Table 2 . Among the assessed pharmaceutics, there were antiplatelet agents, heparins, thrombolysis and bivalirudin. This comparison is presented in Table 3 .

\section{Culprit lesion characteristics}

Also distinguished and compared were the type and frequency of culprit lesion undergoing $\mathrm{PCI}$ in the selected three groups of patients which included de-novo rates, restenosis rates, and instent thrombosis rate. The restenosis rate was also divided into BMS, DES, BRS, DCB and POBA restenosis. In general, the frequency of the de-novo lesions was lower in the SVG group as compared to the non-IMA/SVG group ( $<<0.001)$, and was also lower when compared to IMA group $(p<0.001)$. Whereas, the rate of restenosis was higher in the IMA $(\mathrm{p}=0.02)$ and SVG group $(\mathrm{p}<0.001)$ compared to the non-IMA/SVG group. Moreover, the rate of restenosis in the SVG PCI group was significantly higher when compared to IMA group $(\mathrm{p}<0.001)$. The rate of in-stent thrombosis did not differ significantly between groups. However, it was highest in the SVG PCI group $(0.7 \%)$. All indices are presented in Table 4.

\section{Periprocedural complications}

The periprocedural complications included those which occurred in the operating room and do not cover all complications which took place during subsequent days of hospitalization. the Death rates, cerebral strokes, MI, dissections, no-reflows, puncture site bleedings, allergic reactions and perforations in the non-IMA/SVG group as well as the IMA and SVG groups were assessed. Overall complication rate per patient and per complication in all above-mentioned groups were also compared. Overall complication rates reached $1.92 \%$ when assessed per patient and $2.29 \%$ when assessed per complication in the non-IMA/SVG group of patients and was significantly higher in SVG group compared to non-IMA/SVG group for 
Table 1. Baseline characteristics.

\begin{tabular}{|c|c|c|c|c|c|c|}
\hline Variable & $\begin{array}{c}\text { PCI non- } \\
\text {-IMA/SVG } \\
(n=218,137)\end{array}$ & $\begin{array}{l}\text { PCI IMA } \\
(n=442)\end{array}$ & $\begin{array}{c}\text { P } \\
\text { Non-IMA/ } \\
\text { /SVG vs. IMA }\end{array}$ & $\begin{array}{c}\text { PCI SVG } \\
(n=2,616)\end{array}$ & $\begin{array}{c}\text { P } \\
\text { Non-IMA/ } \\
\text { /SVG vs. SVG }\end{array}$ & $\begin{array}{l}\text { P } \\
\text { SVG vs. } \\
\text { IMA }\end{array}$ \\
\hline Age [years] & $67.0 \pm 10.8$ & $68.9 \pm 9.7$ & $<0.001$ & $70.5 \pm 8.9$ & $<0.001$ & $<0.001$ \\
\hline Gender, males & $147,749(67.7 \%)$ & $339(76.7 \%)$ & $<0.001$ & $2,059(78.7 \%)$ & $<0.001$ & 0.34 \\
\hline Diabetes & $51,720(23.7 \%)$ & $135(30.5 \%)$ & $<0.001$ & $822(31.4 \%)$ & $<0.001$ & 0.71 \\
\hline Hypertension & $154,999(71.0 \%)$ & $337(76.2 \%)$ & 0.01 & $2,079(79.5 \%)$ & $<0.001$ & 0.12 \\
\hline COPD & $5,512(2.5 \%)$ & $11(2.5 \%)$ & 0.95 & $71(2.7 \%)$ & 0.54 & 0.78 \\
\hline Cerebral stroke & $7,156(3.3 \%)$ & $18(4.1 \%)$ & 0.35 & $116(4.4 \%)$ & 0.001 & 0.73 \\
\hline Myocardial infarction & $67,106(30.8 \%)$ & $255(57.7 \%)$ & $<0.001$ & $1,596(61 \%)$ & $<0.001$ & 0.18 \\
\hline $\mathrm{PCl}$ & $80,685(37.0 \%)$ & $240(54.3 \%)$ & $<0.001$ & $1,522(58.2 \%)$ & $<0.001$ & 0.12 \\
\hline Smoking & $42,251(19.4 \%)$ & $45(10.2 \%)$ & $<0.001$ & $278(10.6 \%)$ & $<0.001$ & 0.77 \\
\hline Kidney failure & $11,820(5.4 \%)$ & $36(8.1 \%)$ & 0.01 & $273(10.4 \%)$ & $<0.001$ & 0.13 \\
\hline \multicolumn{7}{|l|}{ Clinical presentation: } \\
\hline Stable angina & $60,356(27.7 \%)$ & $114(25.8 \%)$ & 0.36 & $583(22.3 \%)$ & $<0.001$ & 0.1 \\
\hline Unstable angina & $64,459(29.6 \%)$ & $191(43.2 \%)$ & $<0.001$ & $1,070(40.9 \%)$ & $<0.001$ & 0.36 \\
\hline NSTEMI & $40,371(18.5 \%)$ & $80(18.5 \%)$ & 0.81 & $667(25.5 \%)$ & $<0.001$ & $<0.001$ \\
\hline STEMI & $50,787(23.3 \%)$ & $54(12.2 \%)$ & $<0.001$ & $273(10.4 \%)$ & $<0.001$ & 0.26 \\
\hline Others & $1,833(0.8 \%)$ & $3(0.67 \%)$ & 0.94 & $23(0.9 \%)$ & 0.83 & 0.99 \\
\hline \multicolumn{7}{|l|}{ Vascular access: } \\
\hline AMI & $91,158(41.8 \%)$ & $134(29.9 \%)$ & $<0.001$ & $940(35.9 \%)$ & $<0.001$ & 0.02 \\
\hline Radial right & $125,559(57.6 \%)$ & $47(10.6 \%)$ & $<0.001$ & $524(20.0 \%)$ & $<0.001$ & $<0.001$ \\
\hline Radial left & $35,974(16.5 \%)$ & $149(33.7 \%)$ & $<0.001$ & $661(25.3 \%)$ & $<0.001$ & $<0.001$ \\
\hline Femoral & $55,007(25.2 \%)$ & $237(53.6 \%)$ & $<0.001$ & $1,394(53.3 \%)$ & $<0.001$ & 0.89 \\
\hline \multicolumn{7}{|l|}{ Coronary angiography: } \\
\hline Other & $1,597(0.7 \%)$ & $9(2.0 \%)$ & 0.001 & $37(1.4 \%)$ & $<0.001$ & 0.32 \\
\hline SVD & $137,080(69.2 \%)$ & $235(58.4 \%)$ & $<0.001$ & $1,387(56.8 \%)$ & $<0.001$ & $<0.001$ \\
\hline MVD, LMCA (-) & $51,412(26.0 \%)$ & $128(31.8 \%)$ & 0.007 & $723(29.6 \%)$ & $<0.001$ & 0.37 \\
\hline MVD, LMCA (+) & $7,736(3.9 \%)$ & $38(9.4 \%)$ & $<0.001$ & $326(13.4 \%)$ & $<0.001$ & 0.02 \\
\hline Isolated LMCA & $1,814(0.9 \%)$ & $1(0.2 \%)$ & 0.16 & $4(0.2 \%)$ & $<0.001$ & 0.7 \\
\hline
\end{tabular}

Data given as mean \pm standard deviation or number and percentages. P-value by $\chi^{2}$ test for categorical variables. T-test for continuous variables. AMI - acute myocardial infarction; COPD - chronic obstructive pulmonary disease; LMCA - left-main coronary artery; MVD - multi-vessel disease; NSTEMI - non-ST-segment elevation myocardial infarction; PCI - percutaneous coronary intervention; STEMI — ST-segment elevation myocardial infarction; SVD — single-vessel disease

both estimations $(2.63 \%$ vs. $1.92 \%, \mathrm{p}=0.008$ and $3.09 \%$ vs. $2.29 \%, \mathrm{p}=0.007)$. It did not differ significantly between PCI IMA and non-IMA/ /SVG groups, while the difference was significant between IMA and SVG group (1.58 vs. $3.09, \mathrm{p}=$ $=0.02$ ) for overall complication rate and was lowest in IMA group. The periprocedural mortality rate was the lowest in IMA group compared to the SVG and non-IMA/SVG group, however it did not reach statistical significance $(0.22 \%$ vs. $0.34 \%$ vs. $0.46 \%$, respectively). No-reflows were more frequent in SVG group compared to non-IMA/SVG group $(1.37 \%$ vs. $0.51 \%, \mathrm{p}<0.001)$ and IMA-group (1.37 vs. $0.44, \mathrm{p}=0.01$ ). Also, perforation rate was significantly higher in SVG subgroup compared to non-IMA/SVG PCI group $(0.38 \%$ vs. $0.17 \%, \mathrm{p}=$ $=0.01$ ). There were no perforations noticed in IMA group of patients. No significant differences were noticed in rates of other complications between selected subgroups of patients. The distribution of particular complication rates in selected subgroups is presented in Figure 1A.

\section{Procedural effectiveness}

In IMA group, periprocedural effectiveness assessed as TIMI flow 2-3 after the procedure compared to non-IMA/SVG group ( $96.1 \%$ vs. $96.8 \%$, $\mathrm{p}=0.46$ ), while in SVG group, it was significantly 
Table 2. Procedural variables.

\begin{tabular}{|c|c|c|c|c|c|c|}
\hline Variable & $\begin{array}{c}\text { PCI non- } \\
\text {-IMA/SVG } \\
(\mathrm{n}=218,137)\end{array}$ & $\begin{array}{c}\text { PCI IMA } \\
(n=442)\end{array}$ & $\begin{array}{c}\text { P } \\
\text { Non-IMA/ } \\
\text { /SVG vs. IMA }\end{array}$ & $\begin{array}{c}\text { PCI SVG } \\
(n=2,615)\end{array}$ & $\begin{array}{c}\text { P } \\
\text { Non-IMA/ } \\
\text { /SVG vs. SVG }\end{array}$ & $\begin{array}{c}\text { P } \\
\text { IMA vs. } \\
\text { SVG }\end{array}$ \\
\hline \multicolumn{7}{|l|}{ Type of PCl: } \\
\hline BMS & 7,209 (3.3\%) & $6(1.35 \%)$ & 0.02 & $56(2.14 \%)$ & $<0.001$ & 0.27 \\
\hline DES & $187,042(85.7 \%)$ & $357(80.7 \%)$ & 0.002 & $2,155(82.4 \%)$ & $<0.001$ & 0.4 \\
\hline BRS & $2,925(1.3 \%)$ & $3(0.67 \%)$ & 0.22 & $5(0.19 \%)$ & $<0.001$ & 0.06 \\
\hline DCB & $4,708(2.1 \%)$ & $9(2.0 \%)$ & 0.86 & $117(4.47 \%)$ & $<0.001$ & 0.01 \\
\hline POBA/failed & $16,253(7.4 \%)$ & $67(15.1 \%)$ & $<0.001$ & $282(10.8 \%)$ & $<0.001$ & 0.007 \\
\hline Rotablation & $974(0.4 \%)$ & $0(0 \%)$ & 0.15 & $1(0.03 \%)$ & 0.001 & 0.68 \\
\hline Thrombectomy & $7,001(3.2 \%)$ & $10(2.26 \%)$ & 0.25 & $114(4.35 \%)$ & $<0.001$ & 0.03 \\
\hline Bifurcation & $11,803(5.4 \%)$ & $55(12.4 \%)$ & $<0.001$ & $37(1.41 \%)$ & $<0.001$ & $<0.001$ \\
\hline СTO & $9,235(4.2 \%)$ & $52(11.8 \%)$ & $<0.001$ & $34(1.3 \%)$ & $<0.001$ & $<0.001$ \\
\hline OCT & $392(0.2 \%)$ & $0(0 \%)$ & 0.37 & $8(0.3 \%)$ & 0.13 & 0.24 \\
\hline IVUS & $1,876(0.9 \%)$ & $0(0 \%)$ & 0.05 & $6(0.22 \%)$ & $<0.001$ & 0.31 \\
\hline FFR & $3,248(1.5 \%)$ & $3(0.7 \%)$ & 0.15 & $7(0.26 \%)$ & $<0.001$ & 0.16 \\
\hline Contrast [mL] & $175.6 \pm 78.5$ & $224.3 \pm 108.0$ & $<0.001$ & $217.78 \pm 93.9$ & $<0.001$ & 0.19 \\
\hline Radiation [Gy] & $1,069.5 \pm 963.6$ & $1,204.3 \pm 1048.4$ & 0.005 & $1,216.5 \pm 1058.8$ & $<0.001$ & 0.83 \\
\hline
\end{tabular}

Data given as mean \pm standard deviation or number and percentages. P-value by $\chi^{2}$ test for categorical variables. T-test for continuous variables. BMS - bare-metal stent; BRS — bioresorbable scaffold; CTO — chronic total occlusion; DCB — drug-coated balloon; DES - drug-eluting stent; FFR — fractional flow reserve; IVUS — intravascular ultrasound; OCT — optical coherence tomography; POBA — plain old balloon angioplasty

Table 3. Pharmacological treatment.

\begin{tabular}{|c|c|c|c|c|c|c|}
\hline Medication & $\begin{array}{c}\text { PCI non- } \\
\text {-IMA/SVG } \\
(n=218,137)\end{array}$ & $\begin{array}{l}\mathrm{PCI} \text { IMA } \\
(\mathrm{n}=442)\end{array}$ & $\begin{array}{c}\text { P } \\
\text { Non-IMA/ } \\
\text { /SVG vs. IMA }\end{array}$ & $\begin{array}{c}\text { PCI SVG } \\
(n=2,616)\end{array}$ & $\begin{array}{c}\text { P } \\
\text { Non-IMA/ } \\
\text { /SVG vs. SVG }\end{array}$ & $\begin{array}{c}\text { P } \\
\text { SVG } \\
\text { vs. IMA }\end{array}$ \\
\hline \multicolumn{7}{|c|}{ Pharmacotherapy before $\mathrm{PCI}$} \\
\hline Acetylsalicylic acid & $106,921(49.0 \%)$ & $174(39.4 \%)$ & $<0.001$ & $1,281(49.0 \%)$ & 0.96 & $<0.001$ \\
\hline Unfractionated heparin & $32,414(14.8 \%)$ & $44(9.9 \%)$ & 0.003 & $314(12.0 \%)$ & $<0.001$ & 0.21 \\
\hline LMWH & $2,030(0.9 \%)$ & $1(0.2 \%)$ & 0.12 & $30(1.1 \%)$ & 0.25 & 0.07 \\
\hline \multicolumn{7}{|l|}{$P 2 Y_{12}$ inhibitors } \\
\hline Clopidogrel & $120,066(55.0 \%)$ & $213(48.2 \%)$ & 0.003 & $1,532(58.6 \%)$ & $<0.001$ & $<0.001$ \\
\hline Ticagrelor & $10,988(5.0 \%)$ & $24(5.4 \%)$ & 0.7 & $122(4.7 \%)$ & 0.38 & 0.48 \\
\hline Prasugrel & $1,157(0.5 \%)$ & $2(0.4 \%)$ & 0.82 & $6(0.2 \%)$ & 0.03 & 0.39 \\
\hline GP IIb/IIla inhibitors & $21,066(11.8 \%)$ & $42(11.9 \%)$ & $<0.001$ & $395(17.5 \%)$ & $<0.001$ & 0.008 \\
\hline Bivalirudin & $17(0.007 \%)$ & $0(0 \%)$ & 0.85 & $0(0 \%)$ & 0.65 & - \\
\hline Thrombolysis & $44(0.02 \%)$ & $0(0 \%)$ & 0.74 & $0(0 \%)$ & 0.42 & - \\
\hline \multicolumn{7}{|c|}{ Pharmacotherapy during PCI } \\
\hline Acetylsalicylic acid & $36,977(16.9 \%)$ & $63(14.2 \%)$ & 0.13 & $495(18.9 \%)$ & 0.007 & 0.01 \\
\hline Unfractionated heparin & $183,913(84.3 \%)$ & $359(81.2 \%)$ & 0.07 & $2,309(88.3 \%)$ & $<0.001$ & $<0.001$ \\
\hline LMWH & $8,419(3.8 \%)$ & $22(5.0 \%)$ & 0.22 & $65(2.5 \%)$ & $<0.001$ & 0.003 \\
\hline \multicolumn{7}{|l|}{$P 2 Y_{12}$ inhibitors } \\
\hline Clopidogrel & $91,783(42.1 \%)$ & $181(40.9 \%)$ & 0.63 & $1,124(43.0 \%)$ & 0.35 & 0.42 \\
\hline Ticagrelor & $10,213(4.7 \%)$ & $20(4.5 \%)$ & 0.87 & $126(4.8 \%)$ & 0.74 & 0.79 \\
\hline Prasugrel & $990(0.4 \%)$ & $2(0.4 \%)$ & 0.99 & $14(0.5 \%)$ & 0.53 & 0.82 \\
\hline GP IIb/IIla inhibitors & $836(0.4 \%)$ & $3(0.7 \%)$ & 0.31 & $10(0.4 \%)$ & 0.91 & 0.4 \\
\hline Bivalirudin & $566(0.2 \%)$ & $0(0 \%)$ & 0.28 & $4(0.1 \%)$ & 0.28 & 0.41 \\
\hline Thrombolysis & $344(0.1 \%)$ & $2(0.4 \%)$ & 0.11 & $3(0.1 \%)$ & 0.58 & 0.1 \\
\hline
\end{tabular}

Data given as number and percentage. P-value by $\chi^{2}$ test. GP — glycoprotein; LMWH — low molecular weight heparin 
Table 4. Characteristics of culprit lesion.

\begin{tabular}{|c|c|c|c|c|c|c|}
\hline Variable & $\begin{array}{c}\text { PCI non- } \\
\text {-IMA/SVG } \\
(\mathrm{n}=241,234)\end{array}$ & $\begin{array}{l}\text { PCI IMA } \\
(n=493)\end{array}$ & $\begin{array}{c}\text { P } \\
\text { Non-IMA/ } \\
\text { /SVG vs. } \\
\text { IMA }\end{array}$ & $\begin{array}{c}\text { PCI SVG } \\
(n=2,779)\end{array}$ & $\begin{array}{c}\text { P } \\
\text { Non-IMA/ } \\
\text { /SVG vs. } \\
\text { SVG }\end{array}$ & $\begin{array}{l}\text { P } \\
\text { SVG vs. } \\
\text { IMA }\end{array}$ \\
\hline Number of patients & $218,137(100 \%)$ & $442(0.2 \%)$ & - & $2,616(1.2 \%)$ & - & - \\
\hline Overall lesions count & $241,234(100 \%)$ & $493(0.2 \%)$ & - & $2,779(1.1 \%)$ & - & - \\
\hline De-novo lesions & $228,971(94.9 \%)$ & $459(93.1 \%)$ & 0.06 & $2,378(85.6 \%)$ & $<0.001$ & $<0.001$ \\
\hline Restenosis (overall) & $11,065(4.6 \%)$ & $33(6.7 \%)$ & 0.02 & $380(13.7 \%)$ & $<0.001$ & $<0.001$ \\
\hline Drug-eluting stent restenosis & $7,447(3.1 \%)$ & $25(5.1 \%)$ & 0.01 & $309(11.1 \%)$ & $<0.001$ & $<0.001$ \\
\hline Bare-metal stent restenosis & $3,120(1.3 \%)$ & $4(0.8 \%)$ & 0.34 & $45(1.6 \%)$ & 0.13 & 0.17 \\
\hline Bioresorbable scaffold restenosis & $134(0.05 \%)$ & $1(0.2 \%)$ & 0.16 & $8(0.3 \%)$ & $<0.001$ & 0.73 \\
\hline Drug-coated balloon restenosis & $107(0.04 \%)$ & $1(0.2 \%)$ & 0.09 & $7(0.2 \%)$ & $<0.001$ & 0.83 \\
\hline $\begin{array}{l}\text { Plain-old balloon angioplasty } \\
\text { restenosis }\end{array}$ & $257(0.1 \%)$ & $2(0.4 \%)$ & 0.04 & $11(0.4 \%)$ & $<0.001$ & 0.97 \\
\hline In-stent thrombosis & $1,198(0.5 \%)$ & $1(0.2 \%)$ & 0.35 & $21(0.7 \%)$ & 0.054 & 0.16 \\
\hline
\end{tabular}

Data given as number and percentage. P-value by $\chi^{2}$ test.

lower when compared to non-IMA/SVG group (93.8\% vs. $96.8 \%, \mathrm{p}<0.001)$. The effectiveness of PCI SVG assessed by the TIMI scale was also poorer in PCI SVG group compared to non-IMA/ /SVG PCI group after PCI and separation into TIMI 0 group (5\% vs. $2.25 \%, \mathrm{p}<0.001$ ) and TIMI 4 group (90.3\% vs. $94.1 \%, \mathrm{p}<0.001)$. The TIMI flow distributions in selected groups of patients according to TIMI grade before and after PCI are presented in Figure 1B.

\section{Predictors of selected periprocedural} complications in non-IMA/SVG group

Among significant independent predictors of increased rate of all complications in non-IMA/ /SVG group of patients, the following distinctions were made: age, gender, diabetes, past cerebral stroke, past MI, kidney failure, ACSs, PCI of patients with coronary angiography image other than SVD, femoral access, thrombectomy, RA and cardiac arrest, while among predictors of lower rate of periprocedural complications, also found were: past CABG and patent coronary artery before PCI expressed as TIMI flow 2-3 (Fig. 2A).

Independent predictors of the higher rate of no-reflows in non-IMA/SVG group of patients assessed by multivariable analysis included age, past cerebral stroke, past MI, smoking, hypertension, AMI, other angiographic image than SVD, femoral access and thrombectomy, while among predictors of lower rate of no-reflows patent artery before PCI expressed as TIMI flow 2-3 (Fig. 2B) was also found.
In non-IMA/SVG group of patients, it was found that age, diabetes, past cerebral stroke, past MI, kidney failure, ACSs, other angiographic image than SVD, femoral access, thrombectomy, and cardiac arrest to be among the independent predictors of an increased rate of death, while for predictors of decreased risk of periprocedural death the following was confirmed: male gender, past CABG, hypertension and patent artery before PCI expressed as TIMI flow 2-3 (Fig. 3A).

Among independent predictors of increased risk of procedural perforations in non-IMA/SVG group of patients undergoing PCI assessed in multivariable analysis included the following: age, hypertension, other than single vessel CAD in angiography, PCI of coronary arteries other than LMCA, RA and cardiac arrest, while decreased risk predictors included male gender and TIMI flow 2-3 before PCI (Fig. 3B).

\section{Predictors of selected periprocedural complications assigned to the SVG group}

Considering all complication rates, the multivariable analysis revealed hypertension (odds ratio [OR]: 4.4, 95\% confidence interval [CI]: 1.05-18.5, $\mathrm{p}=0.04)$ and thrombectomy (OR: 3.3, 95\% CI: $1.4-7.6, p=0.005$ ) as predictors of increased rate of all periprocedural complications while among predictors of decreased rate of death, TIMI flow 2-3 before PCI (OR: $0.4,95 \%$ CI: $0.2-0.8, \mathrm{p}=$ $=0.01$ ) in the SVG group was distinguished. Among predictors of increased rate of no-reflows in SVG group assessed by multivariable analysis, these 


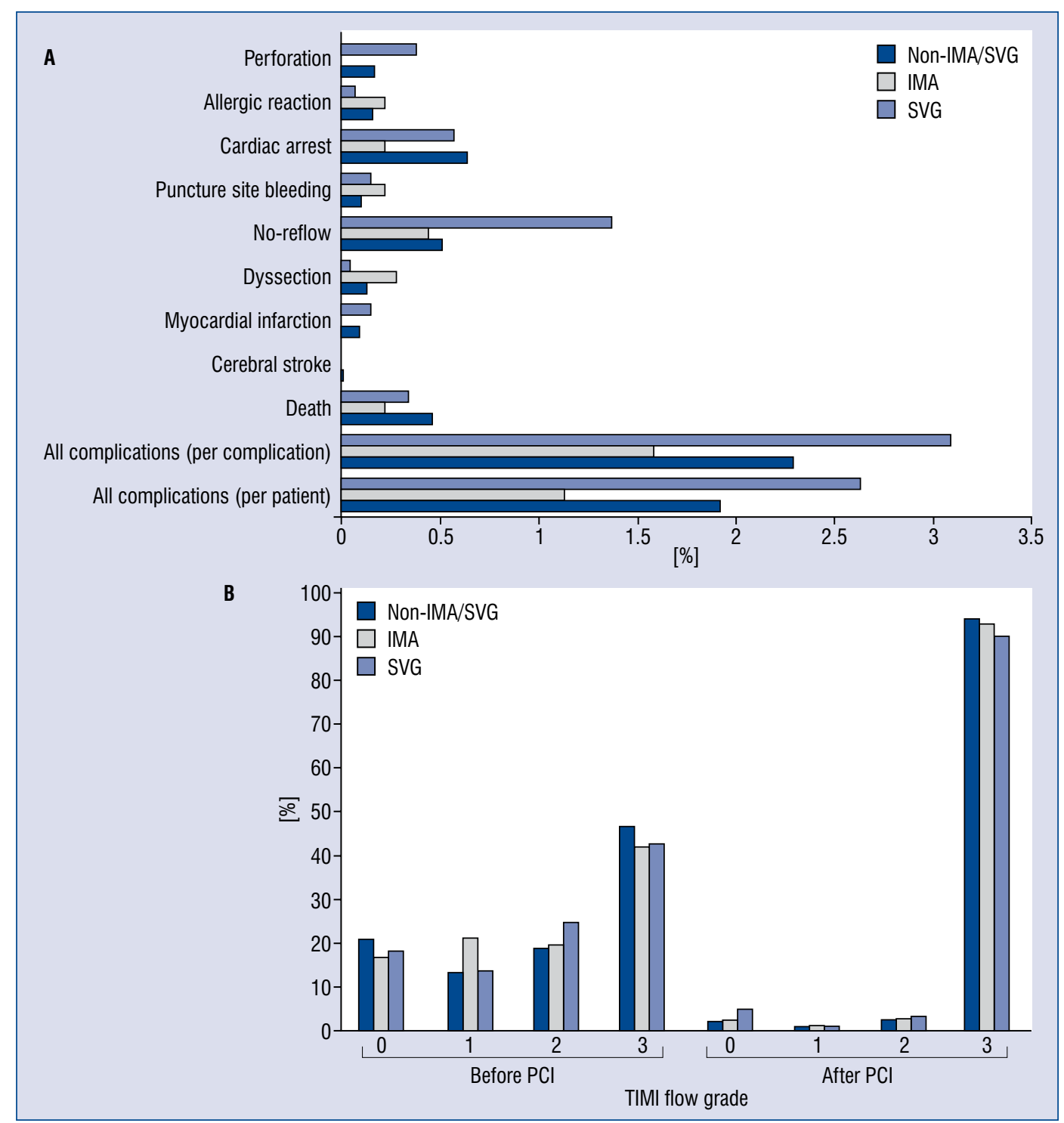

Figure 1. A. The distribution of Thrombolysis in Myocardial Infarction (TIMI) flow grades before and after percutaneous coronary interventions (PCI) expressed as percentages in the non-internal mammary artery (IMA)/saphenous vein grafts (SVG) group, IMA and SVG groups of patients undergoing PCl; B. The distribution of periprocedural complications expressed as percentages in the non-IMA/SVG group, IMA and SVG groups of patients undergoing PCI.

were found: past cerebral stroke (OR: $3.3,95 \%$ CI: $1.0-10.4, \mathrm{p}=0.04)$, ACSs (OR: $5.5,95 \% \mathrm{CI}$ : $1.2-23.9, \mathrm{p}=0.02)$ and thrombectomy (OR: 4.4, $95 \% \mathrm{CI}: 1.7-11.4, \mathrm{p}=0.002)$. Multivariable analysis did not reveal any significant predictors of death in the group of patients undergoing PCI of SVG. The only significant predictor of perforation in the group of patients undergoing PCI of SVG was male gender (OR: 0.17, 95\% CI: 0.04-0.8, $\mathrm{p}=0.02$ ).

\section{Discussion}

The current study confirmed the significant separateness of PCI performed within SVGs when compared to native arteries in terms of several indices such as coronary angiography image, clinical presentation of $\mathrm{CAD}$, culprit lesion characteristics or concomitant diseases, age and gender. This along with many other factors involved, also determines the different panel of predictors of periprocedural complications and their type. The greatest differences in the incidence of periprocedural complications, because of the similarity of PCIs performed on internal mammary arteries to native coronary arteries, were noticed in patients undergoing PCIs of SVGs, and they included an increased rate of all periprocedural complications, no-reflows and perforations. Furthermore, considering significant 


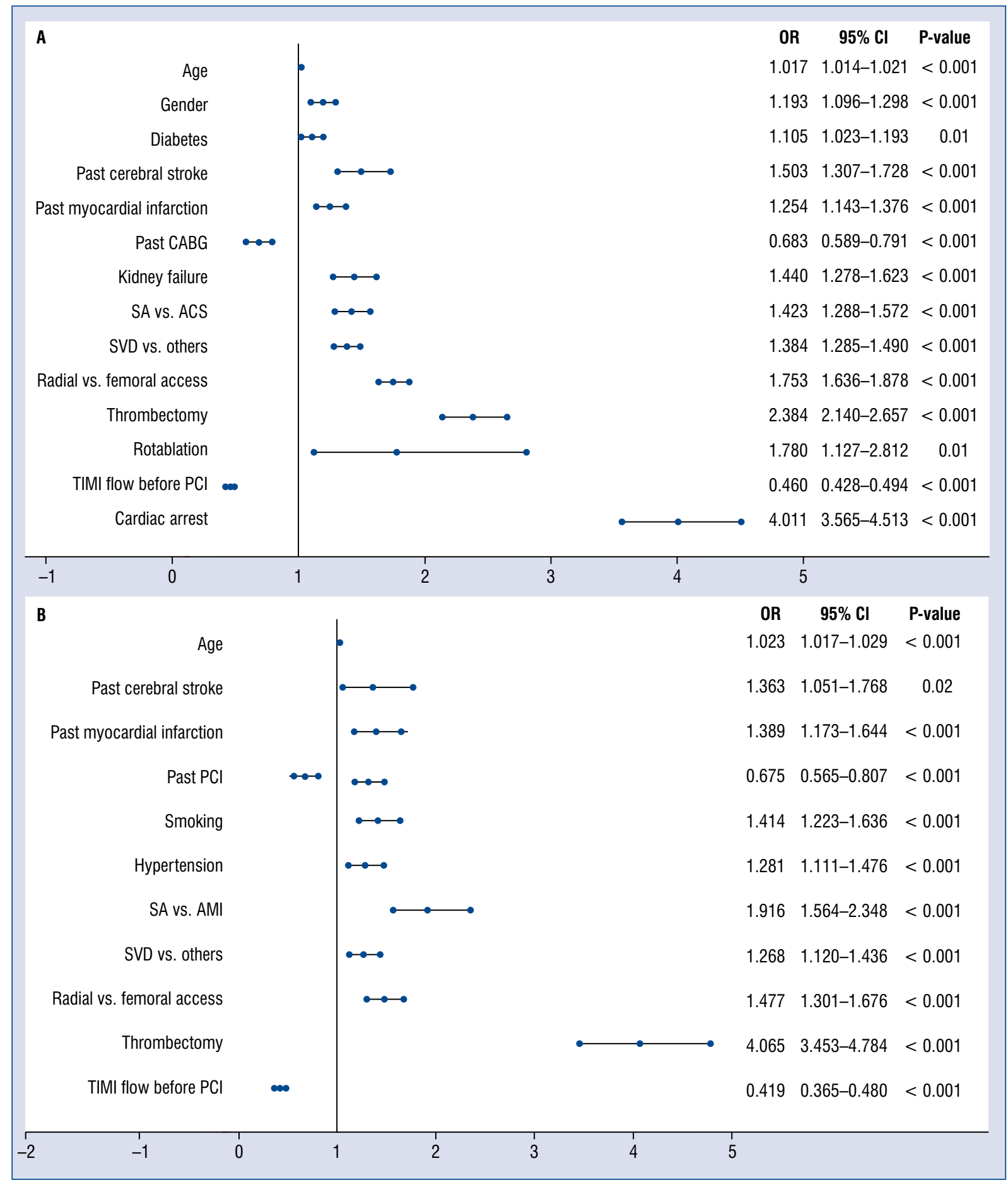

Figure 2. A. Predictors of all periprocedural complications assessed by multivariable analysis in the overall group of patients undergoing percutaneous coronary interventions (PCI); B. Predictors of no-reflows assessed by multivariable analysis in the overall group of patients undergoing PCl; $\mathrm{ACS}$ - acute coronary syndrome; $\mathrm{AMI}$ - acute myocardial infarction; SA — stable angina; CABG — coronary artery bypass grafting; $\mathrm{Cl}$ - confidence interval; OR — odds ratio; SVG — saphenous vein grafts; TIMI — Thrombolysis in Myocardial Infarction.

predictors of periprocedural complications assessed using multivariable analysis, their number was much smaller and was limited to more specific factors among which, and the most deserving of them, included clinical presentation of CAD, TIMI flow before PCI, use of thrombectomy and gender. 


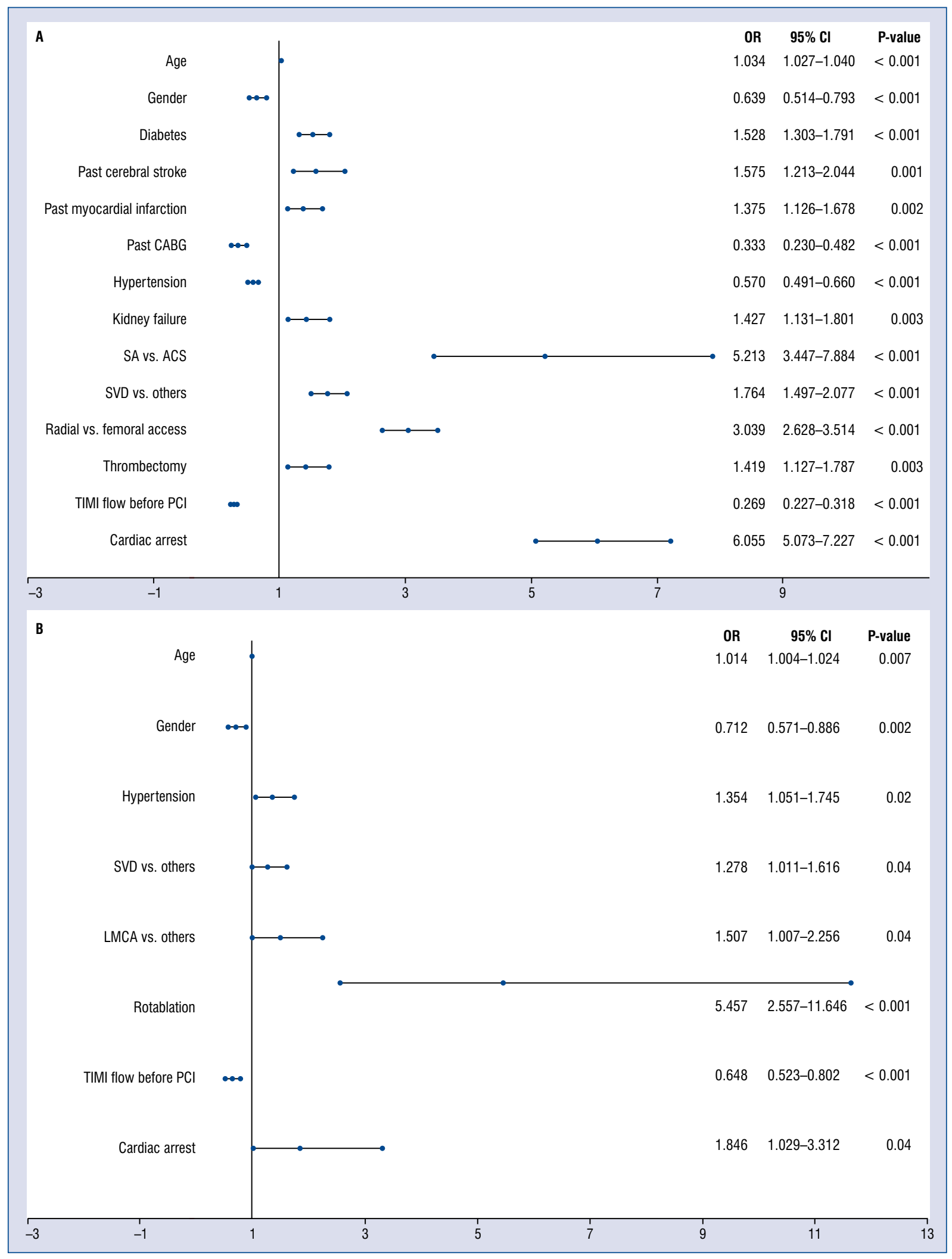

Figure 3. A. Predictors of deaths assessed by multivariable analysis in the overall group of patients undergoing percutaneous coronary interventions (PCI); B. Predictors of perforations assessed by multivariable analysis in the overall group of patients undergoing $\mathrm{PCl}$; $\mathrm{ACS}$ - acute coronary syndrome; $\mathrm{AMI}$ - acute myocardial infarction; $\mathrm{SA}$ - stable angina; CABG — coronary artery bypass grafting; $\mathrm{Cl}$ - confidence interval; LMCA — left-main coronary artery; OR — odds ratio; SVG — saphenous vein grafts; TIMI — Thrombolysis in Myocardial Infarction. 
Considering past $\mathrm{CABG}$ procedure, patients in the IMA and SVG groups are older, more often males and more frequently burdened with an accompanying disease such as diabetes, kidney failure or hypertension. The higher incidence of atherosclerosis risk factors is associated with more advanced atherosclerosis in this selected group of patients. Interestingly, the incidence of chronic obstructive pulmonary disease was similar when comparing all three groups, while there were less smokers in the group of patients undergoing PCI IMA and SVG compared to the non-IMA/SVG group. This could be explained by the fact that CABG procedure is a motivating factor for smoking cessation. Also, the higher rate of peracted MIs and PCIs in IMA and SVG group compared to non-IMA/SVG group is undoubtedly related to the peracted $\mathrm{CABG}$ procedure. A similar relationship may be an explanation for the higher prevalence of MVD in IMA and SVG groups. Similarly, a higher incidence of UA in IMA and SVG groups compared to non-IMA/SVG group, and lower frequency of STEMI and NSTEMI is associated with the presence of a more complicated cardiac vascularization system and associated vascular disorders like the steal syndrome. PCI procedures in patients after $\mathrm{CABG}$ are much more frequently performed with femoral access, due to the fact that radial access in many cases prevents even intubation of the culprit vessel.

Among predictors of periprocedural complication specific for PCI of SVGs, studies published to date have revealed increased intraluminal pressure, graft wall ischemia, thrombosis, fibrin deposition or trauma, secondary repair as well as graft age [11]. For example, Cicek et al. [12] published a study which included 48 patients at a mean age of 62 years, $92 \%$ were men. Indications for revascularization included SA in $71 \%$ of patients, UA in $23 \%$ and AMI in $6 \%$. Stent deployment was performed in all patients. The GP IIb/IIIa inhibitor was used in 56\% patients. No-reflow, defined as TIMI 1 flow, was observed in 2 patients and slow flow, defined as TIMI 2 flow, was observed in 3 patients. Overall, no reflow/slow flow phenomenon occurred in $10 \%$ of patients $(\mathrm{n}=4,2$ patients with $\mathrm{UA}$ and 2 patients with AMI), which was higher compared to the present group. Angiographic success was achieved in $98 \%$ of patients, which was also higher compared to the present group when TIMI flow 2 and 3 was assumed as angiographic success (94\%). All patients survived after stent implantation, but 2 patients experienced non $\mathrm{Q}$-wave $\mathrm{MI}$ and 1 patient experienced $\mathrm{Q}$ wave MI [12]. No relation between no reflow/slow flow and GP IIb/IIIa inhibitor use, hypercholesterolemia or lesion length was found [12]. Another study, which presented procedural results of multiple SVG stenting demonstrated distal embolization in $3.4 \%$ of patients in the single SVG group and in $0.9 \%$ of patients in the multiple SVG group [13]. Furthermore, a study performed on 51 patients who underwent stenting of the bypass graft demonstrated distal embolization in $13.6 \%$ of PCIs [14]. They also reported lower rates of non-Q MIs in patients treated with stent implantation compared to POBA ( $2 \%$ vs. $7 \%$ ). The procedural success rate after SVG stenting in this study was $97 \%$ [15]. Sdringola et al. [16] detected four independent risk factors for the no reflow/slow flow phenomenon as probable thrombus, ACSs, degenerated vein graft and ulcerated plaque. All of this data was not compared, however ACSs were similar predictors of an increased rate of periprocedural no-reflows in patients undergoing PCI of SVG. It was also demonstrated that past cerebral stroke was an independent predictor of increased rate of no-reflows. One possible explanation is that patients after cerebral stroke are usually immobilized to some degree, which decreases hemodynamic response of the cardiovascular system on stress. Consequently, it is related to impaired endothelial response and increased thrombogenicity. The PAMI-2 trial comparing the effectiveness of PCI in patients with grafts and native coronary arteries were revealed among predictors AMI and PCI of native coronary arteries. The procedural success assessed as TIMI flow grade 3 was lower in graft PCI $(70 \%)$ compared to native arteries $(94 \%)$. The reason for this difference was explained by authors as due to extensive thrombus, atherosclerosis burden in bypass grafts and increased rate of distal embolization [17]. However, they did not confirm the relationship between distal embolization and GP IIb/IIIa inhibitor use. Other authors have shown that GP IIb/IIIa inhibitors reduce thrombus burden in SVG lesions and may decrease distal embolization during PCI [18]. In the abovementioned studies, the study group was small and GP IIb/IIIa inhibitor use was limited. They were unable to definitively conclude that GP IIb/IIIa inhibitors reduced distal embolization. Based on our data, univariate analysis confirmed the significant relationship between treatment with GP IIb/IIIa inhibitors before and during $\mathrm{PCI}$ and the rate of periprocedural deaths, no-reflows and all complication counts in the overall group of patients undergoing PCI ( $p<0.001$ in all comparisons). However, multivariable analysis did not confirm such a re- 
lationship. Similar univariate analysis performed in the SVG group revealed only the relationship between no-reflows and overall complication rates for treatment with GP IIb/IIIa inhibitors before PCI, but not after it. Also, multivariable analysis did not confirm those relationships. Large-scale, randomized studies should be performed to clarify this. Another abovementioned study revealed that after stenting of SVG, the lesion length and total cholesterol levels were independent predictors of distal embolization [14]. The present study did not possess this kind of data except for hypercholesterolemia, which was not an independent predictor of periprocedural complications. Kuroda et al. [14] did not observe any no reflow/slow flow during PCI of in-stent restenosis lesions of SVGs. In SVG group from the present study, target lesions were significantly more often restenosis and less frequently de-novo lesions compared to non-IMA/ /SVG group. Univariate analysis did not reveal any relationships between particular periprocedural complications and the presence of restenosis. On the contrary, in-stent thrombosis rate was significantly associated with increased death rate $(\mathrm{p}<$ $<0.001)$ and overall complication rate $(\mathrm{p}=0.04)$ in univariate analysis. In-stent restenosis lesions are pathologically distinct from de novo lesions. In degenerated SVGs, de novo lesions had friable atherogenic material. Bhargava et al. [13] compared two group of patients undergoing PCI of SVG in SVD and MVD groups. The overall angiographic and procedural success rates were similar in both groups. Similarly, major in-hospital complications including death, $\mathrm{Q}$ wave $\mathrm{MI}$ and emergent $\mathrm{CABG}$ were similar in the single stent SVG and multiple stent SVG groups (2.7\% and 2.8\%). However, the periprocedural non $\mathrm{Q}$ wave $\mathrm{MI}$ rate defined as creatinine kinase-MB $>5$ times above the norm was significantly higher in SVG group (28\% vs. $16 \%)$. The frequency of GP IIb/IIIa inhibitor use did not differ significantly between both investigated groups and was higher in the multiple stent group (4\% vs. 6.5\%) [13]. Porto et al. [19] confirmed that the use of post-dilatation was significantly higher in the group of patients who subsequently developed filter no-reflow ( $57 \%$ vs. $26 \%, \mathrm{p}=0.04$ ). In the presented study it was found, that hypertension was an independent predictor of increased rate of overall complications count in SVG group. At the same time it was observed that in the SVG group, hypertension correlated positively with increased rate of no-reflows $(\mathrm{p}=0.02, \mathrm{r}=0.04)$ and cardiac arrests ( $p=0.04, r=0.03)$. This could, at least in part explain this relationship. It was also noticed that the only independent predictor of coronary artery perforation during PCI of SVG was male gender. This is not in line with previously published results performed on the overall group of patients treated with PCI, where female gender was found to be an independent predictor of increased rate of periprocedural perforations [20]. Additionally, in the current study males in SVG group were significantly younger compared to females, which additionally raises some questions.

\section{Limitations of the study}

First of all, this is rather a study based on the nationwide volunteer registry rather than a prospective randomized clinical trial. This tends to decrease and underestimate the detection of periprocedural complication rate and other crucial variables which are dependant on a subjective assessment of the operator, despite a large overall interventional volume included in the present analysis. Furthermore, the current analysis does not include all in-hospital complications which undoubtedly weakens its value. The lack of data evaluating the process of neointimal hyperplasia in the present study, such as intravascular ultrasound or OCT, makes it impossible to assess the relationship between the analyzed risk factors and the type and severity of processes leading to occlusion of evaluated bypasses. Undoubtedly, an advantage of the current study is that the results are closer to real life rather than to randomized clinical trials and shows clinical data depicting the results of SVG PCIs in Central Europe.

\section{Conclusions}

The group of patients undergoing PCI of SVG is at increased risk of periprocedural complications. Among the periprocedural complications typical for PCI of SVG, the following was distinguished: death, no-reflow, perforation and overall periprocedural complication count. Also, the panel of independent predictors of periprocedural complications is different in patients undergoing PCI of SVG compared to non-IMA/SVG, and in the current study, for selected factors it included male gender, ACS, thrombectomy, TIMI flow before PCI, past cerebral stroke and hypertension.

\section{Conflict of interest: None declared}




\section{References}

1. Fitzgibbon GM, Kafka HP, Leach AJ, et al. Coronary bypass graft fate and patient outcome: angiographic follow-up of 5,065 grafts related to survival and reoperation in 1,388 patients during 25 years. J Am Coll Cardiol. 1996; 28(3): 616-626, indexed in Pubmed: 8772748.

2. Węglarz P, Krejca M, Trusz-Gluza M, et al. Neointima development in externally stented saphenous vein grafts. Postepy Kardiol Interwencyjnej. 2016; 12(4): 334-339, doi: 10.5114/ aic.2016.63634, indexed in Pubmed: 27980547.

3. Weintraub WS, Jones EL, Morris DC, et al. Outcome of reoperative coronary bypass surgery versus coronary angioplasty after previous bypass surgery. Circulation. 1997; 95(4): 868-877, indexed in Pubmed: 9054744.

4. Lytle BW, Loop FD, Cosgrove DM, et al. Fifteen hundred coronary reoperations. Results and determinants of early and late survival. J Thorac Cardiovasc Surg. 1987; 93(6): 847-859, indexed in Pubmed: 3494885.

5. Brilakis ES, Rao SV, Banerjee S, et al. Percutaneous coronary intervention in native arteries versus bypass grafts in prior coronary artery bypass grafting patients: a report from the National Cardiovascular Data Registry. JACC Cardiovasc Interv. 2011; 4(8): 844-850, doi: 10.1016/j.jcin.2011.03.018, indexed in Pubmed: 21851896.

6. Selvanayagam JB, Porto I, Channon K, et al. Troponin elevation after percutaneous coronary intervention directly represents the extent of irreversible myocardial injury: insights from cardiovascular magnetic resonance imaging. Circulation. 2005; 111(8): 1027-1032, doi: 10.1161/01.CIR.0000156328.28485.AD, indexed in Pubmed: 15723982.

7. Keeley EC, Velez CA, O'Neill WW, et al. Long-term clinical outcome and predictors of major adverse cardiac events after percutaneous interventions on saphenous vein grafts. J Am Coll Cardiol. 2001; 38(3): 659-665, indexed in Pubmed: 11527613.

8. Januszek R, Siudak Z, Dziewierz A, et al. Predictors of in-hospital effectiveness and complications of rotational atherectomy (from the ORPKI Polish National Registry 2014-2016). Catheter Cardiovasc Interv. 2017 [Epub ahead of print], doi: 10.1002/ ccd.27372, indexed in Pubmed: 29068164.

9. Siudak $Z$, Tokarek T, Dziewierz A, et al. Reduced periprocedural mortality and bleeding rates of radial approach in ST-segment elevation myocardial infarction. Propensity score analysis of data from the ORPKI Polish National Registry. EuroIntervention. 2017; 13(7): 843-850, doi: 10.4244/EIJ-D-17-00078, indexed in Pubmed: 28606891.

10. Windecker S, Kolh P, Alfonso F, et al. 2014 ESC/EACTS Guidelines on myocardial revascularization: The Task Force on Myocardial Revascularization of the European Society of Cardiology
(ESC) and the European Association for Cardio-Thoracic Surgery (EACTS)Developed with the special contribution of the European Association of Percutaneous Cardiovascular Interventions (EAPCI). Eur Heart J. 2014; 35(37): 2541-2619, doi: 10.1093/ eurheartj/ehu278, indexed in Pubmed: 25173339.

11. Higuchi Y, Hirayama A, Shimizu M, et al. Postoperative changes in angiographically normal saphenous vein coronary bypass grafts using intravascular ultrasound. Heart Vessels. 2002; 17(2): 57-60, doi: 10.1007/s003800200044, indexed in Pubmed: 12541095.

12. Cicek D, Doven O, Pekdemir H, et al. Procedural results and distal embolization after saphenous vein graft stenting and angioplasty for in-stent restenosis of grafts. Jpn Heart J. 2004; 45(4): 561-571, indexed in Pubmed: 15353867.

13. Bhargava B, Kornowski R, Mehran R, et al. Procedural results and intermediate clinical outcomes after multiple saphenous vein graft stenting. J Am Coll Cardiol. 2000; 35(2): 389-397, indexed in Pubmed: 10676686.

14. Kuroda Y, Hara K, Nakajima H, et al. Short-term outcome of stent implantation in saphenous vein grafts: predictors of distal embolization and restenosis. Jpn Circ J. 2001; 65(4): 265-270, indexed in Pubmed: 11316120.

15. Savage M, Douglas J, Fischman D, et al. Stent Placement Compared with Balloon Angioplasty for Obstructed Coronary Bypass Grafts. New Engl J Med. 1997; 337(11): 740-747, doi: 10.1056/ nejm199709113371103.

16. Sdringola S, Assali AR, Ghani M, et al. Risk assessment of slow or no-reflow phenomenon in aortocoronary vein graft percutaneous intervention. Catheter Cardiovasc Interv. 2001; 54(3): 318-324, indexed in Pubmed: 11747155.

17. Stone GW, Brodie BR, Griffin JJ, et al. Clinical and angiographic outcomes in patients with previous coronary artery bypass graft surgery treated with primary balloon angioplasty for acute myocardial infarction. Second Primary Angioplasty in Myocardial Infarction Trial (PAMI-2) Investigators. J Am Coll Cardiol. 2000; 35(3): 605-611, indexed in Pubmed: 10716461.

18. Barsness GW, Buller C, Ohman EM, et al. Reduced thrombus burden with abciximab delivered locally before percutaneous intervention in saphenous vein grafts. Am Heart J. 2000; 139(5): 824-829, indexed in Pubmed: 10783216.

19. Porto I, Belloni F, Niccoli G, et al. Filter no-reflow during percutaneous coronary intervention of saphenous vein grafts: incidence, predictors and effect of the type of protection device. EuroIntervention. 2011; 7(8): 955-961, doi: 10.4244/EIJV7I8A151, indexed in Pubmed: 22157481.

20. Fasseas P, Orford JL, Panetta CJ, et al. Incidence, correlates, management, and clinical outcome of coronary perforation: analysis of 16,298 procedures. Am Heart J. 2004; 147(1): 140-145, indexed in Pubmed: 14691432 